\title{
O-Glcnac Glycosylation of Rad51 Plays an Important Role in Promoting Colorectal Cancer Cell Invasion
}

\author{
Qinghua Li ${ }^{1,2}$, Zhuangzhi Cong1, Yongkang Yang ${ }^{1}$, Xinlai Guo ${ }^{1}$, Longjiu Cui ${ }^{1}$, Tiangeng You ${ }^{1 *}$ and Weifeng Tan ${ }^{2}$ \\ ${ }^{1}$ Department of Gastroenterology, Shanghai East Hospital, Tongji University School of Medicine, Shanghai, China \\ ${ }^{2}$ Department of Hepatic Surgery, The Eastern Hepatobiliary Surgery Hospital, Second Military Medical University, Shanghai, China
}

\begin{abstract}
Colorectal cancer (CRC) metastases accounts for two thirds of death cases. Recently, genetic instability has been recognized as one of the hallmarks of metastatic microenvironment. In this study, we identified the relationship between Rad51, a key factor to perform DNA homologous recombination repair, and CRC metastasis, and further at the first time explored the effect of O-GIcNAc glycosylation of Rad51 protein on CRC cells invasion. Generally, the study indicates a novel posttranslational modification of Rad51 protein may play an important regulatory role in the process of CRC cells invasion and represent a potential therapeutic target for clinical management of CRC patients.
\end{abstract}

Keywords: Colorectal cancer; DNA damage repair; O-GlcNAc glycosylation; Rad51; Invasion

\section{Introduction}

CRC is the third most common cancer worldwide, and 30\% of CRC patients have a metastatic disease at the time of diagnosis or relapse after a few months, accounting for the second leading cause of cancer deaths [1]. Notably, 50\% of CRC patients develop liver metastases, responsible for two thirds of death cases, although some therapeutic advances have improved the 5-year survival rates to more than $50 \%$ in recent years [2]. Unfortunately, the pathogenesis of CRC metastases is still not completely understood.

Genetic instability has been recognized as one of the hallmarks of tumor cells. Progressively acquire mutations facilitate tumor cells to invade ambient tissues and metastasize to distant sites; meanwhile accumulated mutations also endue tumor cells resistance to therapeutic inventions [3]. Recent evidence has shown acquired mutations of tumor cells are closely associated with tumor microenvironment; more exposure of tumor cells to hypoxia and acidic microenvironment leads to more genetic instability because of a variety of deregulated DNA repair pathways $[4,5]$. Therefore, understanding the roles of DNA damage repair in the process of tumor metastases may provide insights into developing novel therapeutic strategies.

In eukaryotic cells, Rad51 protein is the key factor to perform DNA homologous recombination repair, and is also closely related to the occurrence and development of many tumors and chemo radiotherapy tolerance [6]. Studies have shown that aberrant expression and nucleotide polymorphisms of Rad51 protein can lead to genomic instability of CRC cells, which is closely related to the high risk of CRC development [7]. However, there are only a few studies on the roles of Rad51 in CRC metastasis. Recently, it has been found that Rad 51 protein expression is regulated by posttranslational modifications including phosphorylation, ubiquitination and SUMO (small ubiquitinlike modifier) [8-10], suggesting that posttranslational modifications play an important regulatory role in the expression of Rad51 protein. Notably, O-linked glycosylation (O-GlcNAc) glycosylation, as a posttranslational modification of functional proteins, has drawn more and more attentions in the process of tumor genesis and metastasis $[11,12]$. Meanwhile, the O-GlcNAc glycosylation will affect the interaction, stability and cell localization of oncoprotein/oncogenic proteins, and then regulate the tumor cells malignant biological behaviors [11]. In this study, we first identified the relationship between Rad51 and CRC metastasis, and further explored the effect of O-GlcNAc glycosylation of Rad51 protein on CRC cells invasion.

\section{Materials and Methods \\ Materials}

Patients and samples: Surgical specimens were obtained from 102 CRC patients, surgically excised from Shanghai East Hospital from August 2012 to August 2016. The histopathological diagnosis of these tumor tissue samples was confirmed as colorectal adenocarcinoma. All patients were not treated with either radiotherapy or chemotherapy before admission. Clinicopathological features and follow-up data of all patients were collected. The human colorectal cancer cell line HT29 was purchased from Shanghai Institutes of Biological Sciences, Chinese Academy of Sciences and cultured in RPMI1640 medium containing $10 \%$ fetal bovine serum at $37^{\circ} \mathrm{C}$ under $5 \% \mathrm{CO}_{2}$. After cells were grown up to $80-90 \%$ confluence, passage was carried out.

Reagents: Immunohistochemistry SP kit was purchased from Beijing Kangwei Century Biotechnology Co., Ltd. Mouse antihuman RAD51 monoclonal antibody (3C10) (MA5-14419) and mouse anti-human O-GlcNAc monoclonal antibody CTD110.6) (sc-59623) were respectively purchased from Thermo Scientific and Santa Cruz Biotechnology. Immunoprecipitation kit and Fast MultiSite Mutagenesis System point mutation kit was respectively purchased from Shanghai Bailey Biotechnology and Beijing Transgen Biotechnology.

\section{Methods}

Immunohistochemistry: IHC procedures in paraffin embedded tissue sections were performed with reference to the manufacturer's instructions. Briefly, sections were incubated with primary antibody

*Corresponding author: Tiangeng You, Department of Gastroenterology, Shanghai East Hospital, Tongji University School of Medicine, Shanghai-200002 China, Tel: +86-15301666812; E-mail: tiangengyou8728@163.com

Received February 06, 2018; Accepted February 12, 2018; Published February 19, 2018

Citation: Li Q, Cong Z, Yang Y, Guo X, Cui L, et al. (2018) O-Glcnac Glycosylation of Rad51 Plays an Important Role in Promoting Colorectal Cancer Cell Invasion. J Biomol Res Ther 7: 160. doi:10.4172/2167-7956.1000160

Copyright: $\odot 2018 \mathrm{Li}$ Q, et al. This is an open-access article distributed under the terms of the Creative Commons Attribution License, which permits unrestricted use, distribution, and reproduction in any medium, provided the original author and source are credited. 
against Rad51 (working dilution ratio was 1: 100) at $4^{\circ} \mathrm{C}$ overnight, after deparaffinization and antigen retrieval. Immunostaining was carried out using a horseradish peroxidase (HRP)-polymer IHC kit. Rad51 expression was found in the nucleus and cytoplasm of CRC cells and evaluated using a immunoreactive score system, integrated with proportion score $(0$, no staining; $1+, \leq 10 \% ; 2+, \leq 35 \% ; 3+, \leq 75 \%$; $4+,>75 \%)$ and intensity score ( 0 , no staining; 1 , weak; 2 , moderate; and 3, strong). Rad51 expression was considered "high" for cases with a total score of $\geq 8$ and "low" for cases $<8$.

Immunoprecipitation (IP): Control or transfected cells were harvested and then lysed on ice in proper IP lysis buffer with protease inhibitors for $30 \mathrm{~min}$, centrifuge at $12,000 \mathrm{~g}$ for $30 \mathrm{~min}$ and then the supernatant was collected and incubated with anti-His antibody and protein $\mathrm{A} / \mathrm{G}$-beads at $4^{\circ} \mathrm{C}$ overnight. After immunoprecipitation, the lysates were centrifuged at $3,000 \mathrm{~g}$ for $5 \mathrm{~min}$ at $4^{\circ} \mathrm{C}$; the supernatant was carefully aspirated and protein A/G-beads washed 3-4 times in $1 \mathrm{ml}$ of lysis buffer, finally, $15 \mu \mathrm{l}$ of $2 \times$ SDS loading buffer was added; after boiled for 10 minutes, SDS-PAGE followed by western blot detection was performed.

Construction and transfection of wild-type pGMLV-His-Rad51 Lentiviral vector and mutants: The wild-type pGMLV-His-Rad51 lentiviral vector was constructed by Jikai Gene Chemical Co., Ltd (Shanghai, China). The wild-type vector was used as a template to introduce the mutation site into the overlapped region using TransStart ${ }^{\circ}$ FastPfu PCR SuperMix. Mutated PCR fragments were spliced together using the special recombinase based on homologous recombination principle in order to achieve multi-point mutation. Multisite-directed mutagenesis of Ser101, Ser102 and Ser107 to Ala101 and Ala102 and Ala107 in Rad51 protein was performed with reference to the reagent manual instructions. Recombinant wild-type lentivirus or mutants were amplified in HEK293T cells and transfected into HT29 cells in Opti-MEM ${ }^{\circledast}$ serum-free media.

Western blot: 'Lentivirus-transfected cells were harvested and homogenized with lysis buffer; then cell lysates were centrifuged at $4^{\circ} \mathrm{C}$ for 30 minutes at $12,000 \mathrm{rpm}$. The quantified supernatant was placed in electrophoresis on a $10 \%$ SDS-polyacrylamide gel and transferred to a $0.2 \mu \mathrm{m}$ PVDF membrane (, Millipore, Billerica, USA) using a Bio-Rad semidry instrument. The membranes were incubated with blocking buffer and followed by subsequent incubation with a primary antibody overnight at $4^{\circ} \mathrm{C}$. After washing, HRP-labeled goat anti-mouse IgG antibody (1:5000, Shanghai Kangcheng Biotechnology Co., Ltd.) was added and incubated for $1 \mathrm{~h}$ at room temperature. ECL western blot system (Super Signal West Pico Chemiluminescent Substrate, Pierce, IL, USA) was used according to the manufacturer's instruction and acquirement and quantification of bands was respectively performed with a ChemiDox XRS imager and Quantity One software (Bio-Rad Laboratories, Inc., Hercules, CA, USA).

CCK8 cell proliferation assay: Cells in the logarithmic phase of growth were trypsinized and centrifuged at $700 \mathrm{rpm}$ for $5 \mathrm{~min}$. After the supernatant was discarded, the cells were resuspended with complete medium to create single cell suspensions with $2 \times 104$ cells $/ \mathrm{mL}$. Cells were seeded into 96 -well plates in $100 \mu \mathrm{L}$ per well with 3 replicate wells and cultured for $24 \mathrm{~h}, 48 \mathrm{~h}, 72 \mathrm{~h}$ and $96 \mathrm{~h}$ respectively. Then $10 \mu \mathrm{L}$ of CCK-8 solution was added to each well and incubate at $37^{\circ} \mathrm{C}$ for $1 \mathrm{~h}$, the plates were analyzed with a microplate reader detection wavelength at $450 \mathrm{~nm}$.

Flow cycles experiment: The procedures of flow cycles experiment was performed according to previous literature [13]. Briefly, Cells in the logarithmic phase of growth were trypsinized. After centrifugation at $800 \mathrm{rpm}$ for $2 \mathrm{~min}$, cells were resuspended and fixed with $3 \mathrm{~mL}$ of $70 \%$ ethanol. $0.5 \mathrm{~mL}$ PI reagent was added and incubated for $15 \mathrm{~min}$ and subjected to BD Biosciences FACSCan to II Flow Cytometer for the detection of cell cycle.

Transwell invasion assay: Mixture of Matrigel and culture media were placed into the bottom of the Transwell chamber with diluted ratio of 1:10 for $30 \mathrm{~min} .1 \times 105$ cells in serum-free medium containing $0.1 \%$ bovine serum albumin were placed into the upper chamber, and $500 \mu$ of complete DMEM media were added to the lower chamber.

24 hours later, the cells remaining in the upper chamber or on the upper membrane were removed with a cotton swab and placed into 24 well plate containing paraformaldehyde for fixation for 30 minutes and treated with $0.25 \%$ crystal violet stain for $10 \mathrm{~min}$ at room temperature prior to washing with water. The number of cells cross to the lower side of the membrane was observed and counted under a microscope.

Statistical analysis: Statistical analysis was done using SPS 16.0 software. The measurement data were analyzed by the $\mathrm{F}$ test its homogeneity of variance, the variance of those who use the t test, the variance of those who use orthostatic $t$ test; comparison of rates between groups using the $\chi 2$ test, the comparison of multiple levels of data using Wilcoxon rank sum test. Data were presented as mean \pm standard deviation; $\mathrm{p}<0.05$ was considered statistically significant.

\section{Results}

\section{Expression of Rad51 in CRC and its correlation with clinical prognosis}

This study recruited 102 patients with CRC, including 64 cases with metastatic lesions and 38 cases without metastatic lesions. The median follow-up time was 16 months (range: 1-41); the follow-up deadline was August 31, 2016. The total positive expression rate of Rad51 was $71.7 \%(73 / 102)$. The positive rate of Rad51 was $81.2 \%(52 / 64)$ in cases with metastatic lesions and $55.3 \%(21 / 38)$ in cases without metastatic lesions, there was a significant difference between the two groups $(\mathrm{p}<0.05)$ (Figure 1A and $1 \mathrm{~B})$. The correlation between Rad51 expression and colorectal cancer clinicopathological parameters was shown in Table 1.

1-year and 3-year overall survival (OS) of 102 CRC patients were $61.9 \%$ and $37.4 \%$, respectively; meanwhile 1 -year and 3 -year progression-free survival rates were $42.6 \%$ and $21.8 \%$ respectively. Among them, for patients with low Rad51 expression, 1-year and 3 -year OS were $63.7 \%$ and $41.3 \%$, respectively, PFS was $90.3 \%$ and $42.6 \%$ respectively; while 1 -year and 3 -year OS of patients with high Rad51 expression were $35.2 \%$ and $15.7 \%$, respectively, and 1 -year and 3 -year PFS was $50.3 \%$ and $21.6 \%$. The survival curves of patients with high and low Rad51 expression were shown in Figure 1C.

\section{O-GIcNAc glycosylation of Rad51 protein in colorectal can- cer cell HT29}

Glycosylation of O-GlcNAc is a newly discovered, dynamic, posttranslational modification of proteins that occurs in the cytoplasm and nucleus, affecting protein structure, regulating protein activity, protein interactions, and protein function [14]. We performed the prediction of potential O-GlcNAc glycosylation sites on the human Rad51 protein (UniProtKB/Swiss-Prot: Q06609) using the O-glycosylation prediction website (http://www.cbs.dtu.dk/services/). There were 9 potential O-GlcNAc glycosylation sites in human Rad51 protein, of which Ser101, Ser102 and Ser107 were most significant (Figure 2A). In addition, we 
Citation: Li Q, Cong Z, Yang Y, Guo X, Cui L, et al. (2018) O-Glcnac Glycosylation of Rad51 Plays an Important Role in Promoting Colorectal Cancer Cell Invasion. J Biomol Res Ther 7: 160. doi:10.4172/2167-7956.1000160

A

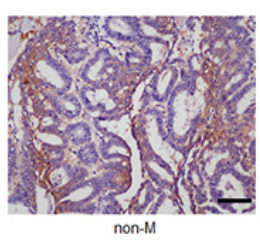

B

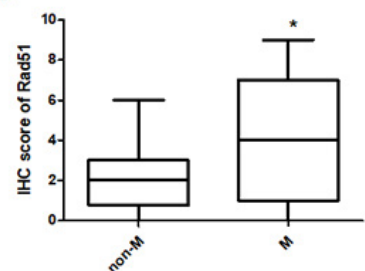

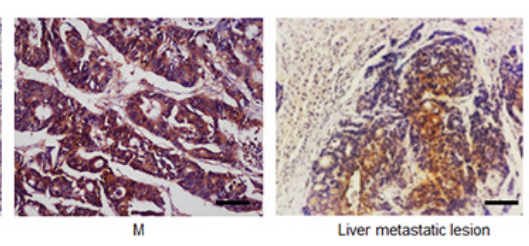

C

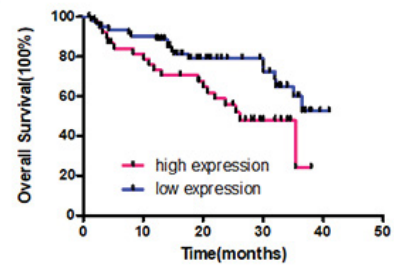

Figure 1: Significant overexpression of Rad51 in CRC tissues with metastatic lesions.

Figure 1A: IHC assays of Rad51 expression in 102 paraffin-embedded CRC tissues. Representative images of CRC tissues without metastasis (left panel) and with metastasis (middle panel) and liver metastatic lesions (right panel) Scale bar: $100 \mu \mathrm{m}$.

Figure 1B: Rad51 expression levels were compared between CRC tissues without metastasis and those with metastasis. Statistical analysis was performed by t-test. ${ }^{*} p<0.05$.

Figure 1C: Kaplan-Meier curve analysis of overall survival in CRC patients by the expression of Rad51. Log-rank test was used to calculate the difference significance.

\begin{tabular}{|c|c|c|c|c|c|}
\hline \multicolumn{6}{|c|}{$\begin{array}{c}\text { Correlation analysis between Rad51 expression and clinicopathological } \\
\text { parameters in patients with CRC }\end{array}$} \\
\hline \multirow{2}{*}{$\begin{array}{l}\text { Clinicopathological } \\
\text { parameters }\end{array}$} & \multirow{2}{*}{ Subsets } & \multirow{2}{*}{ Total no. } & \multicolumn{2}{|c|}{ Rad51 expression } & \multirow{2}{*}{$\boldsymbol{P}$} \\
\hline & & & Low & High & \\
\hline \multicolumn{6}{|l|}{ Age at diagnosis (years) } \\
\hline$\leq 50$ & & 53 & 16 & 37 & 0.621 \\
\hline$>50$ & & 49 & 11 & 38 & \\
\hline \multicolumn{6}{|l|}{ Sex } \\
\hline Male & & 55 & 21 & 34 & 0.566 \\
\hline Female & & 47 & 18 & 29 & \\
\hline \multicolumn{6}{|l|}{ Tumor number } \\
\hline Single & & 58 & 20 & 38 & 0.482 \\
\hline Multiple & & 44 & 17 & 27 & \\
\hline \multicolumn{6}{|l|}{ Differentiation grade } \\
\hline Well & & 30 & 18 & 12 & 0.147 \\
\hline Moderately & & 52 & 22 & 30 & \\
\hline Poor & & 20 & 10 & 10 & \\
\hline \multicolumn{6}{|l|}{ TNM stage } \\
\hline$|-| \mid$ & & 41 & 16 & 25 & 0.005 \\
\hline III-IV & & 61 & 8 & 53 & \\
\hline \multicolumn{6}{|l|}{ Tumor size $(\mathrm{cm})$} \\
\hline$\leq 5$ & & 33 & 13 & 20 & 0.219 \\
\hline$>5$ & & 69 & 25 & 44 & \\
\hline \multicolumn{6}{|l|}{ Depth of invasion } \\
\hline $\mathrm{T} 1+\mathrm{T} 2$ & & 37 & 22 & 15 & 0.008 \\
\hline $\mathrm{T} 3+\mathrm{T} 4$ & & 65 & 27 & 38 & \\
\hline \multicolumn{6}{|l|}{ Distant metastasis } \\
\hline yes & & 38 & 8 & 20 & 0.035 \\
\hline no & & 64 & 24 & 50 & \\
\hline
\end{tabular}

Table 1: Correlation analysis between Rad51 expression and clinicopathological parameters in patients with CRC.
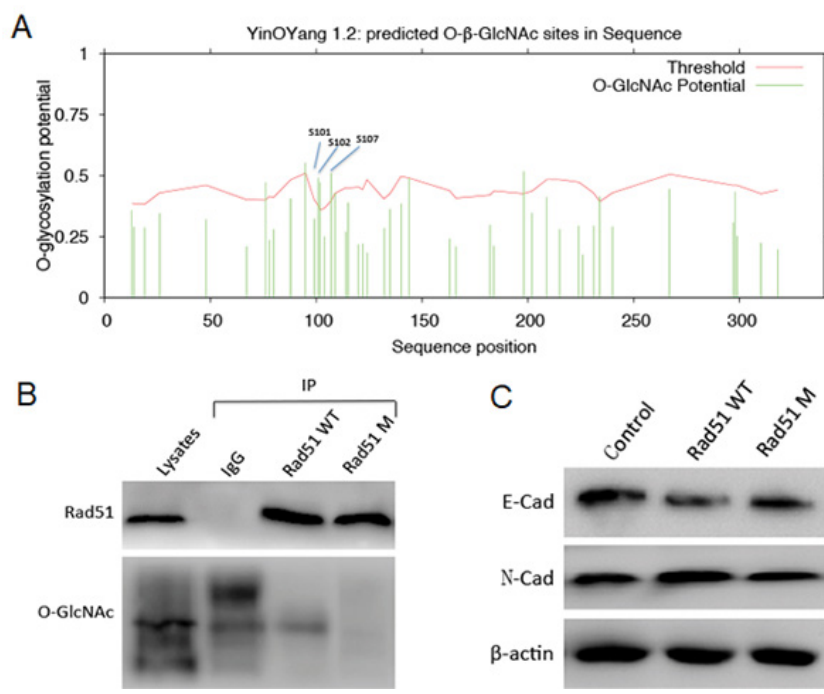

Figure 2: O-GIcNAc Glycosylation of Rad51 Protein in Colorectal Cancer Cell HT29.

Figure 2A: Graphic representation of potential for O-GIcNAc modification in serine and threonine residues in the human Rad51 sequence. Green vertical lines show the potential of S/T residues for O-GICNAc modification and light blue horizontal wavy line shows threshold for modification potential.

Figure 2B: Purified Rad51 proteins from control, Rad51 WT and Rad51 M HT29 cells were detected by western blot analysis with specific anti-O-GlcNAc antibody (CTD110.6).

Figure 2C: Expression of E-cadherin (E-cad) and $\mathrm{N}$-cadherin $(\mathrm{N}$-cad) in control, Rad51 WT and Rad51 M HT29 cells were detected by western blot analysis.

also confirmed the modification by immunoprecipitation followed by western blotting and found O-GlcNAc glycosylation did indeed exist in Rad51 protein in CRC cell line HT29. Furthermore, compared with wild-type Rad51 (WT), the mutant of Rad51 S101A, S102A and S107A (Rad51 M) was not recognized by O-GlcNAc antibody (Figure 2B).

\section{Effect of Rad51 O-GlcNAc glycosylation on invasion ability} of CRC cell line HT29

In order to clarify the influence of Rad51 O-GlcNAc glycosylation on the invasiveness of CRC cell line HT29, we carried out an in vitro Transwell invasion assay. Before experiment, we compared the proliferation of control (transfected negative control), Rad51 WT (transfected wild type Rad51 lentivirus) and Rad51 M (transfected mutant Rad51 lentivirus) cells by using flow cytometry detection and performing a cell proliferation assay. There was no difference in cell proliferation between the three groups (Figure 3A and 3B). In contrast, the result from invasion assay showed that, compared with the control group, the cell invasion ability of Rad51 WT HT29 cells was significantly enhanced; the invasion ability of Rad51 M HT29 cells was partially inhibited (Figure 3C). In addition, we also found that E-cadherin levels and N-cadherin levels were increased in HT29 cells after wild-type Rad51 overexpression, while protein levels of E-cadherin and $\mathrm{N}$-cadherin were both restored after O-GlcNAc glycosylation site mutation (Figure 2C).

\section{Discussion}

Patients with CRC more often died of tumor invasion and metastasis, so clarifying mechanism of CRC invasion and metastasis will help overcome the obstacle and achieve the effective therapy. In recent years, the role of DNA damage repair in tumor development, 


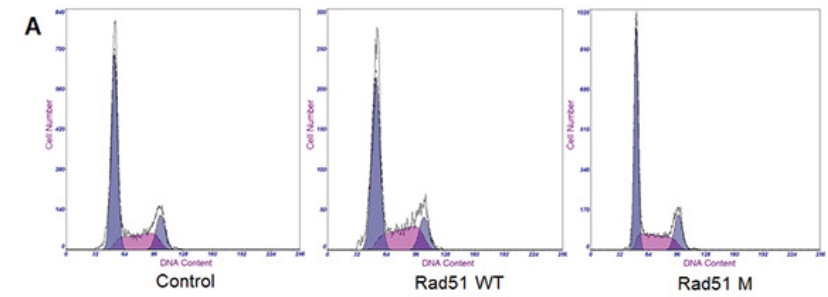

B
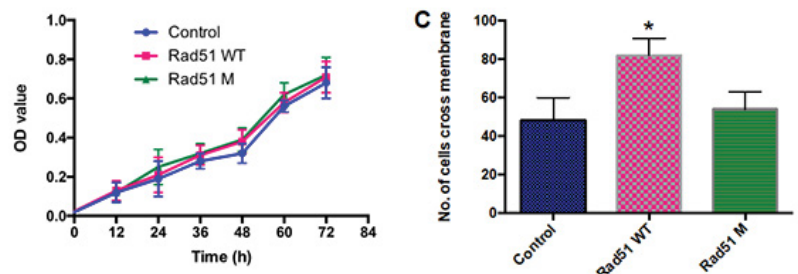

Figure 3: O-GlcNAc glycosylation sites mutation of Rad51 protein inhibited CRC cells invasion in vitro.

Figure 3A: Cell cycle of control, Rad51 WT and Rad51 M was determined with flow cytometry.

Figure 3B: Cell proliferation of control, Rad51 WT and Rad51 M was determined with CCK-8.

Figure 3C: Cell invasion ability in vitro of control, Rad51 WT and Rad51 M was determined with Transwell assay. ${ }^{*} p<0.05$.

diagnosis and treatment has become a hot topic [15]. As a key factor in the repair of DNA double-strand breaks through homologous recombination, the existence of Rad51 is very important for the maintenance of genomic stability. Rad51 overexpression leads to the accumulation of abnormal karyotypes and gene mutations, which are closely related to carcinogenesis and drug resistance [16]. Studies have shown that, such as breast cancer, pancreatic cancer and other tumors overexpressed Rad51 protein, positively correlated to tumor histological grade and stage $[17,18]$. In addition, domestic and foreign researchers also found that gene polymorphism of Rad51 recognized as the risk factor of CRC carcinogenesis. At present, more studies are focusing on the $135 \mathrm{G} / \mathrm{C}$ single nucleotide polymorphism of Rad51 gene, the allelic variation is regarded as a susceptible factor for CRC, but the exact mechanism is not yet clear, which may be related to the presence of a BRCA2 mutation [19]. In contrast, researches on the correlation between Rad51 and tumor metastasis are still insufficient. It is well-known that the process of tumor cell invasion and metastasis is also an immune escape process, during which tumor cells in microenvironment escaped the direct attack of immune cells through enhancing DNA damage repair, meanwhile gained the ability against the cytotoxic effects of chemotherapy drugs finally resulting from acquiring some growth advantage [20]. Recently, a study from the Medical Center of Montpellier University in France revealed the key role of DNA repair in the progression of metastatic circulating colon cancer cells [21]. In this study, we found that Rad51 expression in CRC patients with metastatic lesions was significantly higher than that in CRC patients without metastatic lesions, significantly associated with TNM staging, peritoneal dissemination and lymph node metastasis; at the same time, compared patients with low Rad51 expression, patients with high Rad51 expression had poor prognosis, with lower OS and PFS rates. This result indicated that the high expression of Rad51 is closely related to CRC metastasis, which may be used as a potential predictor or therapeutic target of CRC metastasis.

It is also well-known that the status and types of post-translational modification of proteins determine function, intracellular localization and stability of proteins; meanwhile also confer new chemical targets and biological properties [22]. Common post-translational modifications of proteins include phosphorylation, ubiquitination, acetylation, glycosylation, methylation and SUMOylation, which are involved in the process of tumor cell cycle, proliferation, invasion, apoptosis, adhesion and immune defense, development [23]. O-GlcNAc Glycosylation is an important monosaccharidic modification of serine and threonine residues in mammalian intracellular proteins, which is associated with various diseases [12,24]. In recent years, the role of O-GlcNAc glycosylation has been widely recognized in tumor development and anti-tumor drugs resistance [25]. Studies have shown that increased levels of O-GlcNAc glycosylation of some proteins can enhance the proliferation and metastasis of breast cancer cells [26]; but it also presented cell-specific effects on invasion ability of lung cancer cells and colon cancer cells, which may be related to intracellular lipogenesis and carbohydrate metabolism [27]; furthermore, O-GlcNAc glycosylation can also down-regulate expression of some adhesion factors and up-regulate some protein-degrading enzymes, finally leading to enhance liver cancer cell metastasis [28]. In addition, the specific site O-GlcNAc glycosylation modification of oncoproteins or tumor suppressers can affect protein interaction and stability. For example, O-GlcNAc glycosylation modification of S149 in p53 protein can decrease the interaction between p 53 and ubiquitin ligases, leading to increased stability of p53 protein [29]; after O-GlcNAc glycosylation of 529 amino acid of fructokinase 1 protein can affect tumor cell energy metabolism [30]. Recently, Rad 51 protein ubiquitination can block its binding to BRCA2, thereby affecting cellular homologous recombination [8]. Similarly, our present study also found that one kind of posttranslational modification-O-GlcNAc glycosylation play an important regulatory role in the functional regulation of Rad51 protein in CRC cells. Firstly, we used the O-glycosylation prediction software to analyze potential O-GlcNAc glycosylation sites in human Rad51 protein and found that there are several potential O-GlcNAc glycosylation sites. Subsequently, we applied immunoprecipitation followed by western blotting to confirm the presence of O-GlcNAc glycosylation of Rad51 protein in CRC cells. In addition, no O-GlcNAc glycosylation was detected after mutating three potential modification sites. These results suggested that Rad51 protein can be glycosylated with O-GlcNAc in CRC cells.

Whether does this O-GlcNAc glycosylation modification affect the metastatic ability of CRC cells HT29? To clarify this issue, we firstly detected the effect of wild-type Rad51 and O-GlcNAc glycosylation sites mutant on the proliferation of CRC cell; it was not found that there was significant difference between wild-type and mutant. However, the lack of O-GlcNAc glycosylation can partially inhibit Rad51-mediated invasion, suggesting the existence of O-GlcNAc glycosylation of Rad51 protein was an important factor to promote the invasion of CRC cells, which may be related to epithelial-mesenchymal transition (EMT) of CRC cells, mechanistically due to the down-regulation of E-cadherin and the up-regulation of $\mathrm{N}$-cadherin, explored in our study.

\section{Conclusion}

There are still some limitations in this study. First, confirmation of O-GlcNAc glycosylation of Rad51 protein in CRC cells was performed with monoclonal antibody against O-GlcNAc. Although anti-OGlcNAc (CTD110.6) monoclonal antibody is currently recognized as O-GlcNAc glycosylated detection antibody, it would be more accurate if analyzing O-GlcNAc glycosylation of the protein directly by mass spectrometry. Second, it was observed that, after glycosylation sites mutation of Rad51 protein, E-cadherin and N-cadherin expression changed in CRC cells, but the intrinsic mechanism remains to be 
Citation: Li Q, Cong Z, Yang Y, Guo X, Cui L, et al. (2018) O-Glcnac Glycosylation of Rad51 Plays an Important Role in Promoting Colorectal Cancer Cell Invasion. J Biomol Res Ther 7: 160. doi:10.4172/2167-7956.1000160

Page 5 of 5

further analyzed. Nevertheless, this study is still of great significance. It did not only find at the first time O-GlcNAc glycosylation of Rad51 protein in colorectal cancer cells, but also explored the effect of this novel modification on the function of Rad51 protein, which provides an important key to demonstrate that the posttranslational modifications of proteins in tumor cells play an important regulatory role in the regulation of DNA damage repair; meanwhile display potential clinical translational value. However, the intrinsic mechanism is complicated and still requires further research.

\section{References}

1. Torre LA, Bray F, Siegel RL, Ferlay J, Lortet-Tieulent J, et al. (2015) Global cancer statistics: 2012. CA Cancer J Clin 65: 87-108.

2. De Greef K, Rolfo C, Russo A, Chapelle T, Bronte G, et al. (2016) Multisciplinary management of patients with liver metastasis from colorectal cancer. World $\mathrm{J}$ Gastroenterol 22: 7215-7225.

3. Zhang C, Cao S, Toole BP, Xu Y (2015) Cancer may be a pathway to cell survival under persistent hypoxia and elevated ROS: A model for solid-cancer initiation and early development. Int J Cancer 136: 2001-2011.

4. Broustas CG, Lieberman HB (2014) DNA damage response genes and the development of cancer metastasis. Radiat Res 181: 111-130.

5. Trakselis MA, Seidman MM, Brosh RM Jr (2017) Mechanistic insights into how CMG helicase facilitates replication past DNA roadblocks. DNA Repair 55: 76-82.

6. Pires E, Sung P, Wiese C (2017) Role of RAD51AP1 in homologous recombination DNA repair and carcinogenesis. DNA Repair 599: 76-81.

7. Heinimann K (2013) Toward a molecular classification of colorectal cancer: The role of microsatellite instability status. Front Oncol 31: 3:272.

8. Luo K, Li L, Li Y, Wu C, Yin Y, et al. (2016) A phosphorylation-deubiquitination cascade regulates the BRCA2-RAD51 axis in homologous recombination. Genes Dev 30: 2581-2595.

9. Shima H, Suzuki H, Sun J, Kono K, Shi L, et al. (2013) Activation of the SUMO modification system is required for the accumulation of RAD51 at sites of DNA damage. J Cell Sci 126: 5284-5292.

10. Han D, Liang J, Lu Y, Xu L, Miao S, et al. (2016) Ubiquitylation of Rad51d mediated by E3 Ligase Rnf138 promotes the homologous recombination repair pathway. PLoS One 11: e0155476.

11. Ferrer CM, Sodi VL, Reginato MJ (2016) O-GlcNAcylation in cancer biology: Linking metabolism and signaling. J Mol Biol 428: 3282-3294.

12. Chaiyawat $P$, Netsirisawan $P$, Svasti J, Champattanachai $V$ (2014) Aberran O-GIcNAcylated proteins: New perspectives in breast and colorectal cancer Front Endocrinol 5: 193.

13. Xie J, Liu JH, Liu H, Liao XZ, Chen Y, et al. (2016) Tanshinone IIA combined with adriamycin inhibited malignant biological behaviors of NSCLC A549 cell line in a synergistic way. BMC Cancer 8: 99.
14. Ma Z, Vosseller K (2014) Cancer metabolism and elevated O-GIcNAc in oncogenic signaling. J Biol Chem 289: 34457-65

15. Raschellà G, Melino G, Malewicz M (2017) New factors in mammalian DNA repair-the chromatin connection. Oncogene 36: 4673-4681.

16. Gachechiladze M, Škarda J, Soltermann A, Joerger M (2017) RAD51 as a potential surrogate marker for DNA repair capacity in solid malignancies. Int $J$ Cancer 141: 1286-1294.

17. Godin SK, Sullivan MR, Bernstein KA (2016) Novel insights into RAD51 activity and regulation during homologous recombination and DNA replication. Biochem Cell Biol 94: 407-418.

18. Budke B, Lv W, Kozikowski AP, Connell PP (2016) Recent developments using small molecules to target RAD51: How to best modulate RAD51 for anticancer therapy? ChemMedChem 11: 2468-2473.

19. Toss A, Tomasello C, Razzaboni E, Contu G, Grandi G, et al. (2015) Hereditary ovarian cancer: Not only BRCA 1 and 2 genes. Biomed Res Int.

20. Khan FA, Ali SO (2017) Physiological roles of DNA double-strand breaks. J Nucleic Acids.

21. Alix-Panabières $C$, Cayrefourcq $L$, Mazard $T$, Maudelonde $T$, Assenat $E$, et al. (2017) Molecular portrait of metastasis-competent circulating tumor cells in colon cancer reveals the crucial role of genes regulating energy metabolism and DNA repair. Clin Chem 63: 700-713.

22. Csizmok V, Forman-Kay JD (2017) Complex regulatory mechanisms mediated by the interplay of multiple post-translational modifications. Curr Opin Struct Biol 48: 58-67.

23. Kang C, Lee Y, Lee JE (2016) Recent advances in mass spectrometry-based proteomics of gastric cancer. World J Gastroenterol 22: 8283-8293.

24. Dall'Olio F, Trinchera M (2017) Epigenetic bases of aberrant glycosylation in cancer. Int J Mol Sci 18: 998.

25. Nagel AK, Ball LE (2015) Intracellular protein O-GIcNAc modification integrates nutrient status with transcriptional and metabolic regulation. Adv Cancer Res 126: 137-66.

26. Huang X, Pan Q, Sun D, Chen W, Shen A, et al. (2013) O-GlcNAcylation of cofilin promotes breast cancer cell invasion. J Biol Chem 288: 36418-25.

27. Nanni EA, Huang WR, Hong KH, Ravi K, Fallahi A, et al. (2015) Terahertzdriven linear electron acceleration. Nat Commun 6: 8486 .

28. de Queiroz RM, Carvalho E, Dias WB (2014) O-GIcNAcylation: The sweet side of the cancer. Front Oncol 4: 132

29. Yang WH, Kim JE, Nam HW, Ju JW, Kim HS, et al. (2006) Modification of p53 with $\mathrm{O}$-linked $\mathrm{N}$-acetylglucosamine regulates p53 activity and stability. Nat Cell Biol 8: 1074-1083.

30. YiW, Clark PM, Mason DE, Keenan MC, Hill C, et al. (2012) Phosphofructokinase 1 glycosylation regulates cell growth and metabolism. Science 337: 975-980. 